\title{
SPAG5 is associated with unfavorable prognosis in patients with lung adenocarcinoma and promotes proliferation, motility and autophagy in A549 cells
}

\author{
RUIJIA HUANG and AILI LI \\ Laboratory of Respiratory Diseases, The Affiliated Hospital of Guilin Medical University, Guilin, Guangxi 541001, P.R. China
}

Received November 4, 2019; Accepted July 17, 2020

DOI: $10.3892 /$ etm.2020.9205

\begin{abstract}
Sperm-associated antigen 5 (SPAG5) is involved in the tumorigenesis of multiple cancer types. However, the role of SPAG5 during lung adenocarcinoma (LUAD) progression remains to be fully elucidated. In the present study, the expression of SPAG5 in tumor tissues of patients with LUAD from public cancer databases was analyzed using the online software Gene Expression Profiling Interactive Analysis and University of Alabama Cancer Database. The association of SPAG5 expression levels with the prognosis of patients with LUAD was analyzed using Kaplan-Meier Plotter. In addition, the role of SPAG5 in the LUAD cell line A549 was determined by knocking down its expression with specific small interfering RNA. The results demonstrated that SPAG5 expression was upregulated in LUAD tissues and its high expression was associated with unfavorable prognosis. Furthermore, in A549 cells, SPAG5 promoted proliferation, migration, invasion and autophagy, but inhibited apoptosis. The present results suggest that SPAG5 has an oncogenic role in LUAD and may be a potential prognostic predictor and therapeutic target for LUAD.
\end{abstract}

\section{Introduction}

Lung cancer is the most common cancer type with the highest incidence and mortality among all cancers worldwide (1). In 2018, >2 million new lung cancer cases and 1.8 million lung cancer-associated deaths were estimated to have occurred worldwide (1). Lung cancer is classified into small cell lung carcinoma and non-small cell lung carcinoma (NSCLC), which includes lung adenocarcinoma (LUAD), squamous cell carcinoma and large cell carcinoma, and accounts for $>80 \%$ of lung cancer cases (2). Current treatment options for NSCLC are surgery and adjuvant therapy, including radiation,

Correspondence to: Ms Aili Li, Laboratory of Respiratory Diseases, The Affiliated Hospital of Guilin Medical University, 15 Lequn Road, Guilin, Guangxi 541001, P.R. China

E-mail: 262608860@qq.com

Key words: sperm-associated antigen 5, lung adenocarcinoma, apoptosis, autophagy, motility chemotherapy and targeted therapy (2). Targeted medicines, such as those against epidermal growth factor receptor and anaplastic lymphoma kinase, have been successfully applied in the clinic (3). However, it is still necessary to discover more appropriate molecular targets in order to provide personalized treatment or overcome potential drug resistance.

Sperm-associated antigen 5 (SPAG5, also known as astrin) is involved in mitotic spindle formation and chromosome segregation $(4,5)$, and acts as an important regulator of mitosis and the cell cycle (6). Studies have revealed that SPAG5 has an oncogenic role during the tumorigenesis of multiple cancer types. It was reported to inhibit mTOR complex 1 (mTORC1) signaling to prevent apoptosis in HeLa cells (7), interact with centrosomal protein of $55 \mathrm{kDa}$ to regulate PI3K/AKT signaling, and downregulate scavenger receptor class $\mathrm{A}$ member 5 expression via the $\mathrm{Wnt} / \beta$-catenin pathway in hepatocellular carcinoma cells $(8,9)$, activate the AKT/mTOR pathway to upregulate Wnt3 expression in bladder urothelial carcinoma cells (10), and upregulate survivin expression in gastric cancer (11). It has been indicated that SPAG5 may serve as a prognostic biomarker in cervical cancer (12), breast cancer (13) and bladder urothelial carcinoma (10). A previous study reported that p53 inhibition is essential for the upregulation of SPAG5 in LUAD (14). However, the role and the underlying mechanisms of SPAG5 in LUAD remain to be fully elucidated.

In the present study, SPAG5 expression was assessed in LUAD tissues and the association of SPAG5 expression with the prognosis of patients with LUAD was analyzed. In addition, the role of SPAG5 in cellular proliferation, apoptosis, autophagy and motility of A549 cells was examined.

\section{Materials and methods}

Cell transfection and reagents. The LUAD cell lines A549, HCC827, HCC515, HCC1833, NCI-H1975, NCI-H1993, NCI-H2347 and NCI-H3255 were purchased from the Kunming Cell Bank of the Chinese Academy of Sciences. Cells were maintained in RPMI-1640 medium (Gibco; Thermo Fisher Scientific, Inc.) containing 10\% FBS (Gibco; Thermo Fisher Scientific, Inc.) at $37^{\circ} \mathrm{C}$ in an incubator with $5 \% \mathrm{CO}_{2}$. A549 cells were grown to $\sim 75 \%$ confluence and then transiently transfected with SPAG5 small interfering RNA (siRNA; \#1, 5'-GAGGAAAUUGUAGAGCAUTT-3', \#2, 5'-CCCGACAAC 
UCACAGAGAATT-3', \#3, 5'-CCCGACAACUCACAGAGA ATT-3') or scrambled siRNA (5'-UUCUCCGAACGUGUC ACGUTT-3') (purchased from Shanghai GenePharma Co., Ltd.) at a concentration of $100 \mathrm{pM}$ using Lipofectamine ${ }^{\circledR} 3000$ reagent (Invitrogen; Thermo Fisher Scientific, Inc.) according to the manufacturer's protocols. Bafilomycin (used at a concentration of $100 \mathrm{nM}$ ) was purchased from Cell Signaling Technology, Inc. (cat. no. 54645S).

Western blot analysis. At $48 \mathrm{~h}$ after transfection, total protein was extracted from A549 cells using radioimmunoprecipitation assay lysis buffer (150 mM NaCl; $50 \mathrm{M}$ Tris- $\mathrm{HCl}$; $\mathrm{pH} 7.5$; $1 \%$ Triton $\mathrm{X}-100 ; 0.1 \%$ sodium deoxycholate and $0.1 \%$ SDS) containing $0.1 \%$ proteinase and phosphatase inhibitors (Beijing Solarbio Science \& Technology Co., Ltd.). Immunoblot analysis was then performed as described previously (15). The primary antibodies used were as follows: Rabbit anti-SPAG5 polyclonal antibody [cat.no. 60940; 1:1,800; Cell Signaling Technology,Inc. (CST)], rabbit anti-caspase-3 polyclonal antibody (cat. no. 9662; $1: 1,000 ; \mathrm{CST})$, rabbit anti-cleaved caspase-3 polyclonal antibody (cat. no. 9661; 1:500; CST), rabbit anti-poly(ADP-ribose) polymerase (PARP; cat. no. 9542; 1:1,000; CST), rabbit anti-S6 kinase (cat. no. 2708; 1:1,000; CST), mouse anti-phosphorylated-S6 kinase (cat. no. 9206; 1:1,000; CST), rabbit anti-light chain (LC) 3B polyclonal antibody (cat. no. NB100-2220; 1:1,000; Novus Biologicals, LLC), rabbit anti-p62 polyclonal antibody (cat. no. 39749; $1: 1,000 ;$ CST) and mouse anti- $\beta$-actin monoclonal antibody (cat. no. TA811000; 1:1,000; OriGene Technologies, Inc.). The secondary antibodies used were as follows: Horseradish peroxidase (HRP)-conjugated anti-rabbit immunoglobulin G (IgG; cat. no. 7074; 1:5,000; CST) and HRP-conjugated anti-mouse IgG (cat. no. 7076; 1:5,000; CST). Densitometric analysis was performed using FluorChem SA 6.0.0 (Informer Technologies, Inc.).

Reverse transcription-quantitative PCR (RT-qPCR). Total RNA was isolated from A549 cells using TRIzol ${ }^{\circledR}$ reagent (Thermo Fisher Scientific, Inc.) according to the manufacturer's protocol. RNA concentration was measured using a NanoDrop ${ }^{\mathrm{TM}} 2000$ spectrophotometer (Thermo Fisher Scientific, Inc.). A total of $2 \mu \mathrm{g}$ RNA was reverse transcribed into complementary DNA using a reverse transcription kit (cat. no. KR103-04; Tiangen Biotech Co., Ltd.) according to the manufacturer's protocol. qPCR was performed using SYBR ${ }^{\circledR}$ Premix Ex Taq ${ }^{\mathrm{TM}}$ II (Takara Bio, Inc.) to detect SPAG5 gene expression. GAPDH was amplified in parallel as the internal control. The gene-specific primers used were as follows: SPAG5 forward, 5'-CATCTCACAGTGG GATAACTAATAAAC-3' and reverse, 5'-CAGGGATAGGTG AAGCAAGGATA-3' and GAPDH forward, 5'-CAGGAGGCA TTGCTGATGAT-3' and reverse, 5'-GAAGGCTGGGGCTCA TTT-3'. The following thermocycling conditions were used for the qPCR: Pre-denaturation at $95^{\circ} \mathrm{C}$ for $30 \mathrm{sec}$, followed by 40 cycles of denaturation at $95^{\circ} \mathrm{C}$ for $5 \mathrm{sec}$, annealing at $60^{\circ} \mathrm{C}$ for $20 \mathrm{sec}$ and extension at $72^{\circ} \mathrm{C}$ for $10 \mathrm{sec}$, and final extension at $72^{\circ} \mathrm{C}$ for $30 \mathrm{sec}$. The $2^{-\Delta \Delta \mathrm{Cq}}$ method was used to calculate relative gene expression (16).

Flow cytometric analysis. A549 cells $\left(1 \times 10^{5}\right.$ cells/well) were seeded in 12-well plates and cultured for $17 \mathrm{~h}$. Subsequently, the cells were transfected with SPAG5 siRNA or control
siRNA for $48 \mathrm{~h}$. The cells were harvested and incubated with $5 \mu \mathrm{l}$ FITC Annexin $\mathrm{V}$ and $5 \mu \mathrm{l}$ propidium iodide (PI) for $15 \mathrm{~min}$ in the dark, and $300 \mu 11 \mathrm{X}$ binding buffer was then added. Finally, the cells were analyzed by flow cytometry (Muse ${ }^{\mathrm{TM}}$ Cell Analyzer; Merck Millipore). Cells with positive FITC Annexin V and negative PI were considered to be apoptotic.

Cell proliferation assay. A549 cells were transfected with SPAG5 siRNA or control siRNA. At $48 \mathrm{~h}$ post-transfection, $5 \times 10^{3}$ cells were seeded into 96-well plates in RPMI-1640 medium containing $2 \%$ FBS. The media was replaced every day for 4 days. Cell proliferation was determined with an MTT kit (cat. no. M1020; Beijing Solarbio Science \& Technology Co., Ltd.) according to the manufacturer's protocol. In brief, MTT was dissolved in $0.1 \mathrm{MPBS}$ at a concentration of $5 \mathrm{mg} / \mathrm{ml}$. Subsequently, $10 \mu \mathrm{l}$ MTT solution was added to each well, followed by incubation at $37^{\circ} \mathrm{C}$ for $4 \mathrm{~h}$. Finally, RPMI-1640 culture medium was discarded and $200 \mu \mathrm{l}$ dimethyl sulfoxide was then added to each well to completely dissolve the crystals. The absorbance at a wavelength of $490 \mathrm{~nm}$ was measured with a microplate reader (SpectraMax ${ }^{\circledR}$ Plus 384; Molecular Devices).

Cell migration and invasion assay. Following transfection with SPAG5 siRNA or negative control for $48 \mathrm{~h}, 8-\mu \mathrm{m}$ pore size culture inserts (Transwell; Costar; Corning Inc.) were used for the migration assay and Matrigel Invasion Chambers (Transwell; Costar; Corning Inc.) were used for the invasion assay. The inserts were placed into the wells of 24-well culture plates, separating the plates into upper and lower chambers. A total of $600 \mu 1$ RPMI-1640 medium containing 20\% FBS was added to the lower chambers. Furthermore, $3 \times 10^{4}$ cells in $200 \mu \mathrm{l}$ serum-free RPMI-1640 medium were added to each upper chamber. After incubation at $37^{\circ} \mathrm{C}$ in an atmosphere with $5 \% \mathrm{CO}_{2}$ for $24 \mathrm{~h}$, non-invading or non-migrating cells were removed from the top wells with a cotton swab. Cells that had transgressed to the bottom of the membrane were fixed with $4 \%$ paraformaldehyde for $20 \mathrm{~min}$ at room temperature and stained with $0.1 \%$ crystal violet overnight at $4^{\circ} \mathrm{C}$. Randomly selected images of three independent fields of each well were captured with a phase-contrast microscope (magnification, x100; Olympus TH4-200; Olympus Corporation) and the cells were manually counted.

Colony formation assay. Following transfection with SPAG5 siRNA or negative control for $48 \mathrm{~h}, 6 \times 10^{2}$ cells were seeded into each well of six-well plates, and $2 \mathrm{ml} \mathrm{RPMI-1640}$ culture medium was added into each well. Cells were cultured at $37^{\circ} \mathrm{C}$ in a humidified incubator with $5 \% \mathrm{CO}_{2}$ for $10-15$ days. Cells was washed with PBS three times, fixed with $4 \%$ neutral paraformaldehyde solution at room temperature for $30 \mathrm{~min}$, followed with three PBS washes, and $2 \mathrm{ml} 1 \%$ crystal violet solution was added to each well and incubated at room temperature for $2 \mathrm{~h}$. The cells were washed three times with PBS after the crystal violet solution was discarded. Once the plates were dry, the colonies were counted and images were captured.

Analysis of SPAG5 expression in LUAD tissues. LUAD data from The Cancer Genome Atlas (TCGA) were analyzed using the Gene Expression Profiling Interactive Analysis (GEPIA; gepia.cancer-pku.cn) online tool (17). The Okayama Lung cohort (GSE31210 in Gene Expression Omnibus database) was 

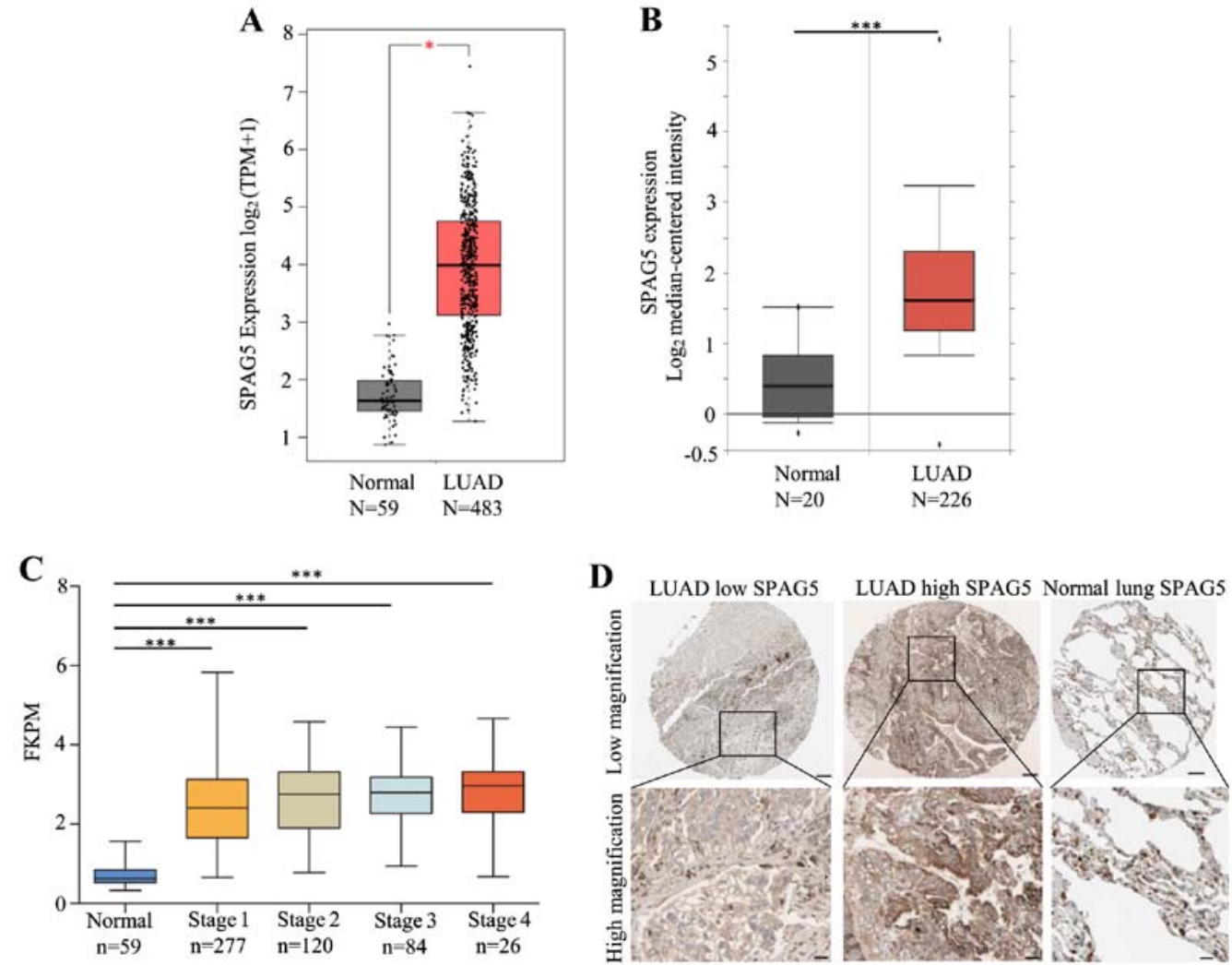

Figure 1. SPAG5 expression is upregulated in LUAD. (A) SPAG5 expression in LUAD tissues ( $\mathrm{n}=483$ ) and normal lung tissues ( $\mathrm{n}=59)$ from TCGA database was analyzed with the Gene Expression Profiling Interactive Analysis online software. The results demonstrated that SPAG5 expression was significantly increased in LUAD. "P<0.05. (B) SPAG5 expression in LUAD tissues $(n=226)$ and normal lung tissues $(n=20)$ from the ONCOMINE database (Okayama Lung) was analyzed online. The results demonstrated that SPAG5 expression was significantly increased in LUAD. ${ }^{* * *} \mathrm{P}<0.001$. (C) SPAG5 expression in different stages of LUAD was significantly increased compared with in normal tissues. However, there was no significant difference between any stages. SPAG5 expression at different stages (stage 1, $n=277$; stage 2, $n=120$; stage 3, $n=84$; stage 4, $n=26)$ and normal lung tissues $(n=59)$ from TCGA database was analyzed. One-way ANOVAs and Tukey's post hoc test were used for the comparison between the groups. ${ }^{* * *} \mathrm{P}<0.001$. (D) Immunohistochemistry staining results from The Human Protein Atlas database demonstrated that SPAG5 expression was upregulated in LUAD tissues. The patient ID for the sample with low expression was 2585 and that for high expression was 3391. Scale bar, $100 \mu \mathrm{m}$ in low-magnification images and $25 \mu \mathrm{m}$ in high-magnification images. TCGA, The Cancer Genome Atlas; LUAD, lung adenocarcinoma; SPAG5, sperm-associated antigen 5; TPM, transcripts per million; FPKM, fragments per kilobase of transcript per million mapped reads.

analyzed using ONCOMINE (www.oncomine.org) $(18,19)$. TCGA LUAD datasets (TCGA Pan-Cancer Atlas) were downloaded from cbioportal (https://www.cbioportal.org/datasets) and SPAG5 expression at different clinical stages of TCGA LUAD was analyzed using GraphPad Prism 5.0 software (GraphPad Software, Inc.) (20). Comparisons among normal groups and different clinical stages were performed using one-way ANOVA followed by Tukey's post hoc test. SPAG5 expression data by immunohistochemical (IHC) staining were obtained from The Human Protein Atlas (HPA) database (www.proteinatlas.org) (21).

Prognosis analysis. The univariate analysis of SPAG5 expression on overall survival (OS), first progression (FP) and post-progression survival (PPS) was analyzed using the online tool Kaplan Meier plotter (www.kmplot.com) (22). The cut-off values were as follows: 266 for OS, 216 for FP and 305 for PPS, which were the best threshold values automatically determined by the software. Hazard ratio (HR), 95\% confidence intervals (CI) and log-rank P-values were calculated using Cox regression. The log-rank test was used to evaluate the differences between survival curves. Cox proportional hazards model was used for multivariate analysis of sex, American Joint Committee on Cancer (AJCC) stage $\mathrm{T}$ and stage $\mathrm{N}$, smoking history and SPAG5 expression on OS and FP. $\mathrm{P}<0.05$ was considered to indicate a statistically significant difference.

Statistical analysis. SPSS 13.0 software (SPSS, Inc.) was used to perform statistical analysis. Values are expressed as the mean \pm SD. Comparisons between two groups were performed using Student's t-test and comparisons among multiple groups were performed using one-way ANOVA followed by Tukey's post hoc test. All experiments were performed at least three times independently and representative data are presented. The log-rank test was used to evaluate the differences between survival curves. Cox proportional hazards model was used for multivariate analysis. $\mathrm{P}<0.05$ was considered to indicate a statistically significant difference.

\section{Results}

SPAG5 expression is upregulated in LUAD tissues and its high expression is associated with unfavorable prognosis. To determine SPAG5 expression in LUAD tissues, the LUAD data in TCGA were analyzed using the online tool GEPIA (17). The results indicated that SPAG5 expression was significantly upregulated in LUAD tissues $(n=483)$ compared with normal lung tissues $(n=59)(P<0.05$; Fig. 1A). To confirm the TCGA 
results, SPAG5 expression was analyzed in LUAD tissues $(n=226)$ and normal lung tissues $(n=20)$ in the Okayama Lung cohort from the ONCOMINE database $(18,19)$. The results demonstrated that SPAG5 expression was significantly increased in LUAD compared with normal lung tissues $(\mathrm{P}<0.001$; Fig. 1B), consistent with the results of the LUAD cohort from TCGA. Furthermore, SPAG5 expression we analyzed in LUAD samples at different clinical stages from TCGA. The results showed that SPAG5 expression at stages 1-4 (stage 1, $n=277$; stage $2, n=120$; stage $3, n=84$; stage 4, $\mathrm{n}=26$;) was significantly increased compared with normal lung tissues $(\mathrm{n}=59)(\mathrm{P}<0.001$; Fig. $1 \mathrm{C})$, while there was no significant difference between any two stages of LUAD. Since the TCGA and ONCOMINE data were obtained at the RNA level, alterations in SPAG5 expression were further analyzed at the protein level by searching the HPA database (21). The results of the HPA included two datasets for IHC staining of lung cancer tissue (HPA022008 and HPA022479) comprising a total of 11 cases stained with one of two different SPAG5 antibodies. The HPA022008 dataset comprised one LUAD sample with low and five with medium SPAG5-positive staining, while the HPA022479 dataset comprised two LUAD samples with negative, two with low and one with highly positive SPAG5 staining. Representative images are provided in Fig. 1D. The IHC data confirmed SPAG5 expression in LUAD tissues at the protein level.

To reveal the potential prognostic value of SPAG5 in patients with LUAD, the association between SPAG5 expression and OS, FP and PPS was analyzed using the online tool Kaplan Meier plotter (22). The results of the univariate analysis demonstrated that high expression of SPAG5 is associated with unfavorable OS and FP (Fig. 2A and B), while there was no significant association with PPS (Fig. 2C), suggesting that SPAG5 expression may serve as a predictor of OS and FP. The results of the multivariate analysis of AJCC stage, sex and smoking history suggested that high expression of SPAG5 is an independent prognostic indicator for OS and FP (Table I).

Knockdown of SPAG5 suppresses the proliferation, migration and invasion of A549 cells. To investigate the role of SPAG5 in lung cancer in vitro, SPAG5 protein expression was examined in multiple LUAD cell lines by western blot analysis. The results indicated SPAG5 was expressed in all cell lines tested and its expression in A549 cells was the highest (Fig. 3A). Therefore, the A549 cell line was selected for subsequent experiments. A total of three siRNAs against SPAG5 were designed and their effects to suppress SPAG5 expression were assessed by RT-qPCR and western blot analysis. A549 cells were individually transfected with SPAG5 siRNAs\#1-3 and control siRNA, and SPAG5 expression was examined $48 \mathrm{~h}$ after transfection. The results indicated that all SPAG5 siRNAs significantly reduced SPAG5 expression at the RNA and protein level compared with control siRNA transfection, and SPAG5 siRNA\#2 had the most potent inhibitory effect (Fig. 3B). Thus, SPAG5 siRNA\#2 was used in further experiments.

Since SPAG5 was indicated to be associated with unfavorable OS and FP of patients with LUAD, it was hypothesized that SPAG5 may have a role in the regulation of proliferation
Table I. Cox regression multivariate analysis on OS and FP.

\begin{tabular}{llcc}
\hline Rate & \multicolumn{1}{c}{ Variable } & Hazard ratio (95\% CI) & P-value \\
\hline OS & Sex & $1.55(0.86-2.79)$ & 0.1410 \\
& AJCC stage T & $2.64(1.34-5.21)$ & 0.0051 \\
& AJCC stage N & $2.83(1.69-4.75)$ & 0.0001 \\
& Smoking history & $0.82(0.38-1.77)$ & 0.6138 \\
& SPAG5 & $1.89(1.01-3.53)$ & 0.0469 \\
FP & Sex & $1.22(0.66-2.26)$ & 0.5333 \\
& AJCC stage T & $2.87(1.34-6.17)$ & 0.0068 \\
& AJCC stage N & $2.48(1.41-4.37)$ & 0.0017 \\
& Smoking history & $1.41(0.7-2.84)$ & 0.3412 \\
& SPAG5 & $2.05(1.05-4)$ & 0.0357 \\
\hline
\end{tabular}

OS, overall survival; FP, first progression; AJCC, American Joint Committee on Cancer.

and motility of LUAD cells. To test this hypothesis, the proliferation and motility of A549 cells transfected with SPAG5 siRNA were analyzed using MTT and Transwell assays, respectively. The results suggested that, compared with that in the control siRNA group, the proliferation (Fig. 4A), migration (Fig. 4B) and invasion (Fig. 4C) of A549 cells transfected with SPAG5 siRNA was significantly attenuated.

Knockdown of SPAG5 induces apoptosis in A549 cells. The effects of SPAG5 knockdown on A549 cell apoptosis were then examined by flow cytometry and western blot analysis. Flow cytometric analysis demonstrated that SPAG5 siRNA led to significant increase in apoptotic cells compared with control siRNA (Fig. 5A). To confirm the flow cytometry results, the activation of the apoptotic markers caspase- 3 and PARP was detected by western blot analysis. The result suggested that, compared with the control siRNA group, transfection of SPAG5 siRNA resulted in a decrease of caspase- 3 and PARP protein and a simultaneous increase of cleaved caspase-3 and cleaved PARP protein (Fig. 5B), indicating that SPAG5 knockdown induced apoptosis in A549 cells.

Knockdown of SPAG5 inhibits autophagy in A549 cells. As a previous study indicated that in HeLa cells, SPAG5 inhibits mTORC1, which is a vital upstream negative regulator of autophagy (7), it was hypothesized that SPAG5 may enhance autophagy. To assess this hypothesis, A549 cells were transfected with SPAG5 siRNA for $48 \mathrm{~h}$ and then treated with the autophagy inhibitor bafilomycin for $1 \mathrm{~h}$. Subsequently, the autophagic markers LC3 and p62 were examined by western blot analysis. The results suggested that transfection of SPAG5 siRNA led to a decrease of LC3-II and a simultaneous increase of p62 compared with control siRNA (Fig. 6A), indicating that SPAG5 knockdown inhibited autophagy. In addition, mTORC1 activity was examined by detecting phosphorylation of its substrate S6K by western blot analysis. The results indicated that compared with the control siRNA group, S6K phosphorylation increased following SPAG5 knockdown (Fig. 6B), implying increased mTORC1 activity. 

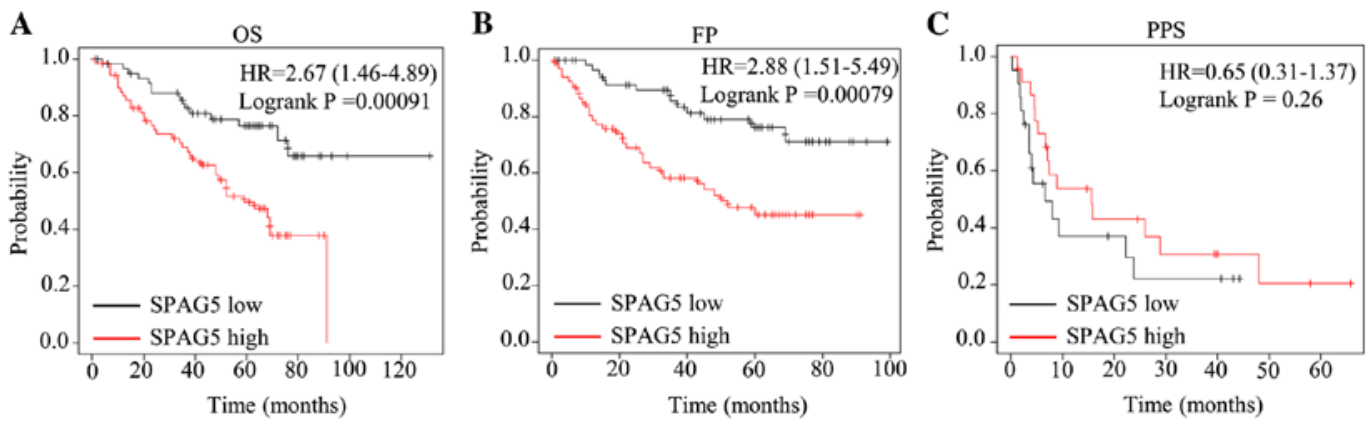

Figure 2. High SPAG5 expression is associated with unfavorable OS and FP in patients with LUAD. (A) High SPAG5 expression was associated with unfavorable OS. The OS curve of 360 patients was plotted [cut-off value, 266; HR (95\% CI)=2.04 (1.38-3.01); log-rank P=0.00024]. (B) High SPAG5 expression was associated with unfavorable FP. The FP curve of 170 patients was plotted [cut-off value, 216; HR (95\% CI) $=2.6(1.37-4.93) ; \log$-rank P=0.0023]. (C) High SPAG5 expression was not significantly associated with PPS. The PPS curve of 125 patients was plotted [cut-off value, $305 ; \mathrm{HR}(95 \% \mathrm{CI})=1.49(0.86-2.61)$ $\log$-rank $\mathrm{P}=0.15]$. The online tool Kaplan Meier Plotter was used to draw the graphs. OS, overall survival; FP, first progression; LUAD, lung adenocarcinoma; SPAG5, sperm-associated antigen 5; HR, hazard ratio; PPS, post-progression survival.

A

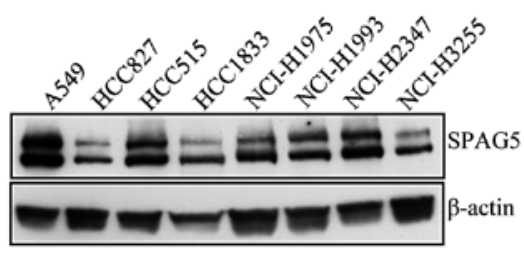

B

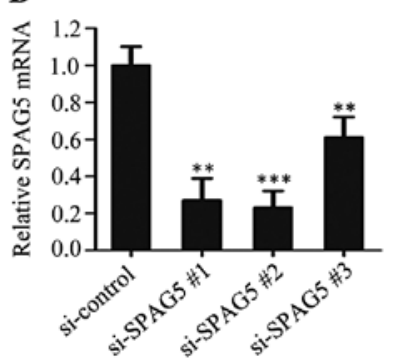

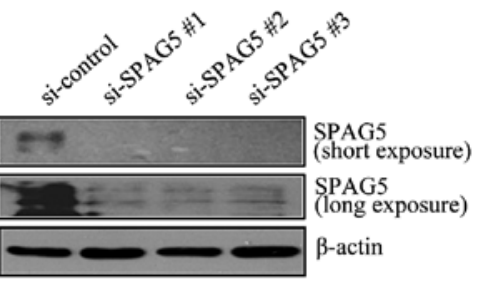

Figure 3. SPAG5 is expressed in multiple lung adenocarcinoma cell lines. (A) SPAG5 protein levels were determined by western blot analysis. The results showed that A549 cells had the highest SPAG5 expression among the cell lines tested. (B) A549 cells were treated with SPAG5 siRNA for $72 \mathrm{~h}$ and SPAG5 mRNA (left) and protein (right) levels were then examined. The results indicated that SPAG5 siRNA \#2 had the highest efficacy. ${ }^{* *} \mathrm{P}<0.01$ and ${ }^{* * * *} \mathrm{P}<0.001$ vs. si-control. SPAG5, sperm-associated antigen 5; siRNA, small interfering RNA.

A

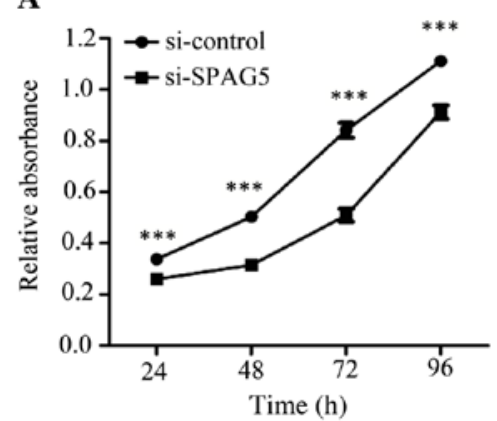

B

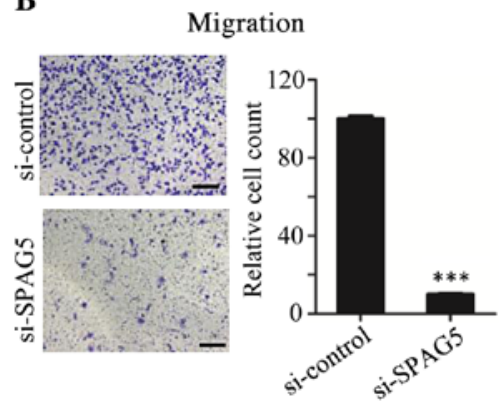

C

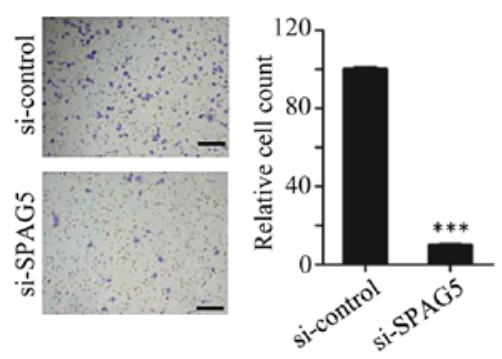

D

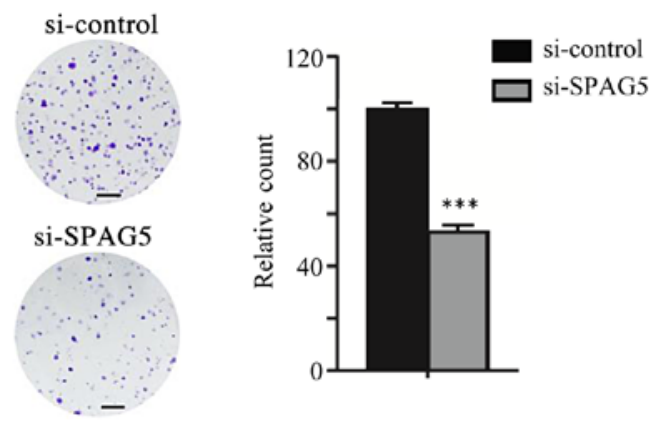

Figure 4. SPAG5 knockdown inhibits proliferation, migration, invasion and colony formation of A549 cells. (A) A549 cells were transfected with SPAG5 siRNA and the proliferation was then determined by MTT assay. (B) Migration and (C) invasion of A549 cells transfected with siRNA was measured using a Transwell assay without or with Matrigel, respectively. Representative images of filter membranes are shown. Magnification, x200. Scale bar, $200 \mu \mathrm{m}$. (D) Colony formation assay results of A549 cells transfected with SPAG5 siRNA or control siRNA. Scale bar, $5 \mathrm{~mm}$. ${ }^{* * *} \mathrm{P}<0.001$ vs. si-control. SPAG5, sperm-associated antigen 5; siRNA, small interfering RNA. 

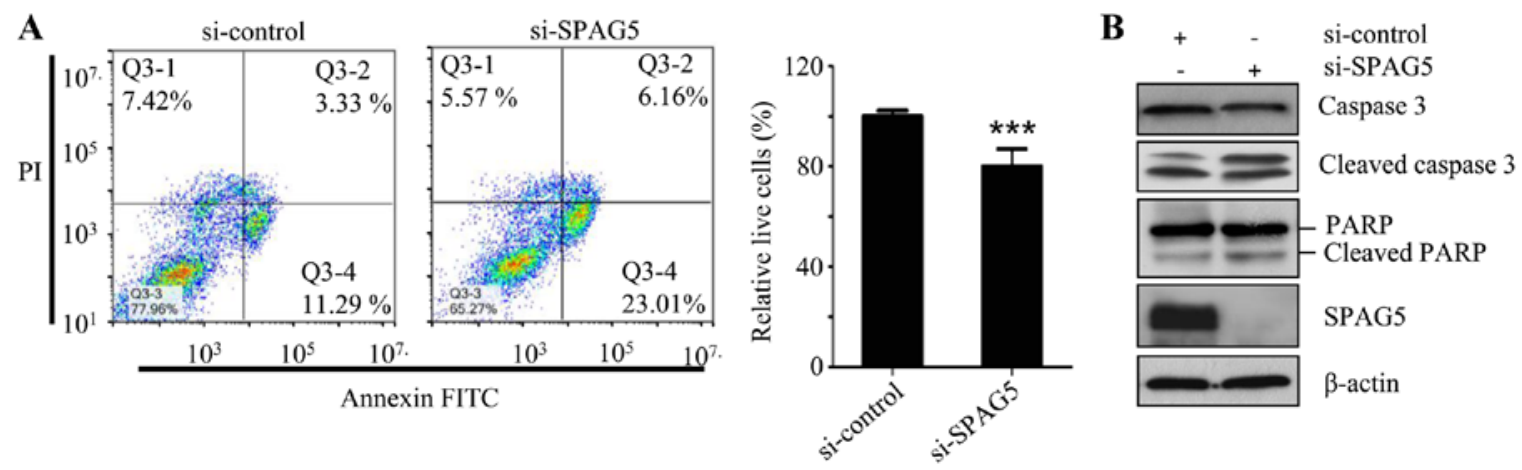

Figure 5. SPAG5 knockdown induces apoptosis. A549 cells were transfected with SPAG5 siRNA for $72 \mathrm{~h}$. (A) Apoptosis was detected by flow cytometry. Representative flow cytometry dot plots (Annexin $\mathrm{V}^{+} / \mathrm{PI}$ cells were considered apoptotic) and quantitative results are shown. (B) The apoptotic markers caspase-3 and cleaved caspase-3 were detected by western blot analysis. Representative western blot images are shown. Data are expressed as the mean \pm standard deviation. ${ }^{* * *} \mathrm{P}<0.001$ vs. si-control. SPAG5, sperm-associated antigen 5; siRNA, small interfering RNA; PI, propidium iodide; PARP, poly (ADP-ribose) polymerase.
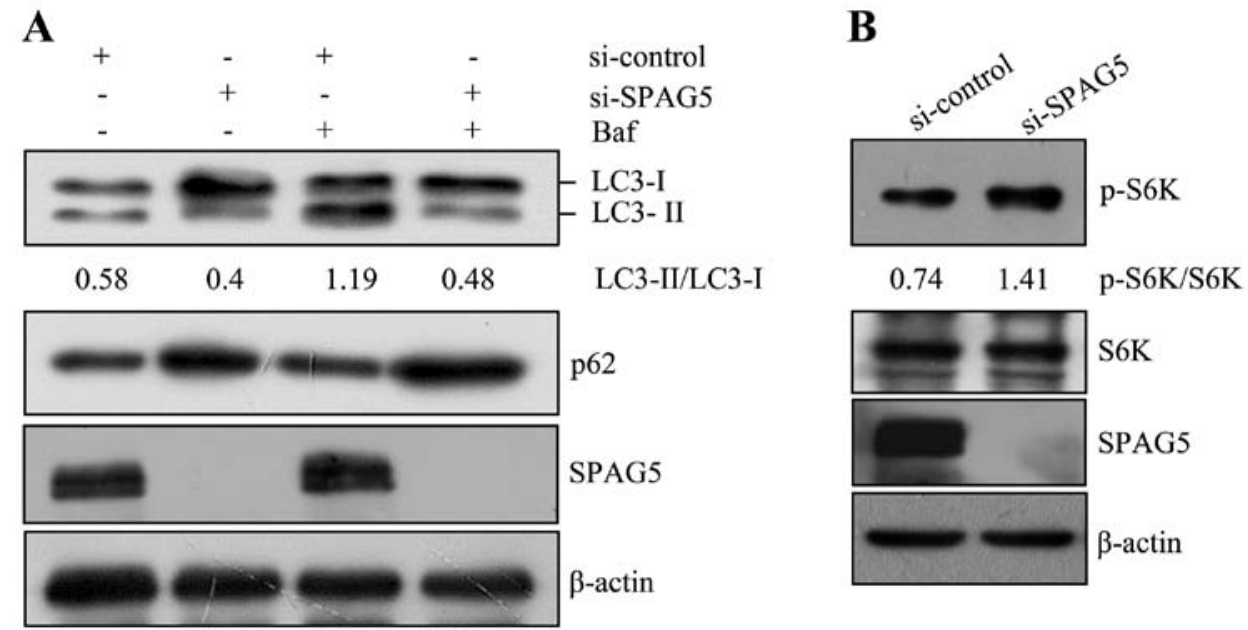

Figure 6. SPAG5 knockdown suppresses autophagy by activating mTORC1. A549 cells were transfected with SPAG5 siRNA for 48 h, followed by bafilomycin treatment for $1 \mathrm{~h}$. The autophagic markers LC3 and p62 and S6K phosphorylation were detected by western blot analysis. (A) The levels of LC3-II were reduced and p62 protein levels were increased by SPAG5 siRNA, implying inhibition of autophagy. Bafilomycin treatment did not result in any obvious accumulation of LC3. (B) Phosphorylation of S6K was increased by SPAG5 siRNA, implying increased mTORC1 activity. SPAG5, sperm-associated antigen 5; siRNA, small interfering RNA; LC3, light chain 3; mTORC1, mTOR complex 1, Baf, bafilomycin.

\section{Discussion}

Patients with lung cancer have a poor prognosis and the 5-year survival is $<18 \%$ (2). Therefore, it is urgent to discover novel biomolecules that may be used as diagnostic markers and/or therapeutic targets. Välk et al (23) analyzed the gene expression profiles of 81 tissue samples of patients with NSCLC, revealing that SPAG5 is upregulated in NSCLC and therefore suggested that SPAG5 may be a biomarker in this cancer type. However, the underlying mechanisms of SPAG5 in LUAD have remained elusive. The present study demonstrated that SPAG5 expression was upregulated in LUAD by analyzing its expression in two different large LUAD cohorts from two databases. However, SPAG5 expression did not change significantly from LUAD stage 1 to stage 4 . These results indicated that SPAG5 upregulation occurs early during the tumorigenesis of LUAD, implying that SPAG5 may be a potential diagnostic marker for LUAD. In addition, high expression of SPAG5 was indicated to be associated with unfavorable OS and FP, which may also suggest that SPAG5 may be used as a prognostic marker in LUAD.
Dysregulation of proliferation is a hallmark of malignant tumor growth (24). It was hypothesized that inhibiting SPAG5 expression may suppress certain cancerous features of LUAD cells. Thus, this possibility was tested in vitro using LUAD A549 cells, which exhibited high endogenous SPAG5 expression. The present study demonstrated that, in A549 cells, knockdown of SPAG5 inhibited cell proliferation and induced apoptosis, indicating that SPAG5 may have a role in the regulation of cell proliferation and death. These results are in agreement with those of previous studies on cervical cancer (12), bladder urothelial carcinoma (10) and gastric cancer (11). Although the apoptosis of A549 cells was analyzed upon SPAG5 knockdown by flow cytometry to detect annexin and by western blotting to examine the apoptotic markers caspase-3 and PARP, it would be useful to confirm the results in cells treated with caspase inhibitor when performing further experiments. Certain carcinomas arise from epithelial tissues and may subsequently obtain the capability of migration and invasion to progress to higher pathological grades of malignancy due to aberrations in the expression of relevant genes associated with invasion and metastasis, such as N-cadherin (24). The present study 
indicated that knockdown of SPAG5 in A549 cells inhibited cell migration and invasion, suggesting that SPAG5 may have a role in tumor metastasis. As SPAG5 may act via the Wnt $/ \beta$-catenin and AKT/mTOR pathways $(8,10)$, both of which are crucial pathways in a variety of cancers, it is necessary to explore which mechanism enables LUAD cells to obtain those features in the future. As epidermal growth factor receptor (EGFR) acts upstream of the AKT/mTOR pathway, it was questioned whether there is any association between EGFR and SPAG5. To address this, the correlation between EGFR and SPAG5 expression in LUAD tissues was analyzed with GEPIA. However, a weak but not significant correlation was observed (data not shown), indicating they do not significantly affect the expression of each other.

Autophagy is a lysosomal degradation pathway through which cells are able to recycle unwanted or damaged proteins or organelles to maintain cellular homeostasis or in response to microenvironmental stress (25). Accumulating evidence has indicated that autophagy may serve as a tumor-suppressing or tumor-promoting process, depending on the context or tumor type (26). A previous study reported that SPAG5 inhibits mTORC1 activity by binding to Raptor to prevent mTORC1 assembly in HeLa cells (7). Given that mTORC1 acts as a suppressor of autophagy, it was hypothesized that SPAG5 may have a role in the regulation of autophagy. Thus, the effect of SPAG5 knockdown on autophagy was examined in the present study. The results indicated that knockdown of SPAG5 inhibited autophagy accompanied by increased S6K phosphorylation, implying SPAG5 promoted autophagy through regulating the activity of mTORC1 in A549 cells. Therefore, SPAG5 may promote tumor cell survival through enhancing autophagy to recycle cellular components in the microenvironment lacking oxygen and nutrients. To the best of our knowledge, the present study was the first to reveal the role of SPAG5 in the regulation of autophagy.

Since high SPAG5 expression was observed in LUAD tissues and may contribute to cancerous features, including excessive proliferation, evasion of apoptosis and enhancement of autophagy, it is important to uncover how SPAG5 expression is regulated in LUAD. Wang et al (14) observed that SPAG5 expression in LUAD cells is suppressed by p53. Song et al (27) reported that microRNA (miR)-1179 inhibited SPAG5 expression in NSCLC cells. Wang et al (28) revealed that long non-coding RNA LINC00875 may sponge miR-1179 to regulate SPAG5 expression in LUAD cells. As the in vitro experiments in the present study were only performed in A549 cells with siRNA to knock down SPAG5, further experiments including knockdown and overexpression of SPAG5 should be performed in other LUAD cell lines in order to confirm the conclusions.

In summary, the present study demonstrated that the expression of SPAG5 was upregulated in LUAD tissues and its high expression is associated with unfavorable clinical outcomes. It may promote the proliferation, motility and autophagy, and inhibit apoptosis of LUAD cells. The present results indicated that SPAG5 has an oncogenic role in LUAD and may be a potential prognostic predictor and therapeutic target for LUAD.

\section{Acknowledgements}

Not applicable.

\section{Funding}

This work was supported in part by the Research Enhancement Project for Junior Faculty in Higher Education Institutes of Guangxi (grant no. 2019KY0522), the Scientific Research Project for Junior Faculty in Guilin Medical College (grant no. 2018glmcy055) and the Open Research Fund from Guangxi Key Laboratory of Liver Injury and Repair Molecular Medicine (grant no. GXLIRMMKL-201816).

\section{Availability of data and materials}

The datasets used and/or analyzed during the current study are available from the corresponding author on reasonable request.

\section{Authors' contributions}

AL conceived the study. RH performed the bioinformatical analysis and AL performed the experiments. RH and AL analyzed and interpreted the data. AL and RH wrote the manuscript. All authors read and approved the final manuscript.

\section{Ethics approval and consent to participate}

Not applicable.

\section{Patient consent for publication}

Not applicable.

\section{Competing interests}

The authors declare that they have no competing interests.

\section{References}

1. Bray F, Ferlay J, Soerjomataram I, Siegel RL, Torre LA and Jemal A: Global cancer statistics 2018: GLOBOCAN estimates of incidence and mortality worldwide for 36 cancers in 185 countries. CA Cancer J Clin 68: 394-424, 2018.

2. Zappa C and Mousa SA: Non-small cell lung cancer: Current treatment and future advances. Transl Lung Cancer Res 5: 288-300, 2016.

3. Herbst RS, Morgensztern D and Boshoff C: The biology and management of non-small cell lung cancer. Nature 553: 446, 2018.

4. Mack GJ and Compton DA: Analysis of mitotic microtubule-associated proteins using mass spectrometry identifies astrin, a spindle-associated protein. Proc Natl Acad Sci USA 98: 14434-14439, 2001.

5. Thein KH, Kleylein-Sohn J, Nigg EA and Gruneberg U: Astrin is required for the maintenance of sister chromatid cohesion and centrosome integrity. J Cell Biol 178: 345-354, 2007.

6. Liu L, Akhter S, Bae JB, Mukhopadhyay SS, Richie CT, Liu X and Legerski R: SNM1B/Apollo interacts with astrin and is required for the prophase cell cycle checkpoint. Cell Cycle 8: 628-368, 2009.

7. Thedieck K, Holzwarth B, Prentzell MT, Boehlke C, Kläsener K Ruf S, Sonntag AG, Maerz L, Grellscheid SN, Kremmer E, et al: Inhibition of mTORC1 by astrin and stress granules prevents apoptosis in cancer cells. Cell 154: 859-874, 2013.

8. Yang YF, Zhang MF, Tian QH, Fu J, Yang X, Zhang CZ and Yang H: SPAG5 interacts with CEP55 and exerts oncogenic activities via PI3K/AKT pathway in hepatocellular carcinoma. Mol Cancer 17: 117, 2018.

9. Liu H, Hu J, Wei R, Zhou L, Pan H, Zhu H, Huang M, Luo J and Xu W: SPAG5 promotes hepatocellular carcinoma progression by downregulating SCARA5 through modifying $\beta$-catenin degradation. J Exp Clin Cancer Res 37: 229, 2018. 
10. Liu JY, Zeng QH, Cao PG, Xie D, Yang F, He LY, Dai YB, Li JJ, Liu XM, Zeng HL, et al: SPAG5 promotes proliferation and suppresses apoptosis in bladder urothelial carcinoma by upregulating Wnt 3 via activating the $\mathrm{AKT} / \mathrm{mTOR}$ pathway and predicts poorer survival. Oncogene 37: 3937-3952, 2018.

11. Liu G, Liu S, Cao G, Luo W, Li P, Wang S and Chen Y: SPAG5 contributes to the progression of gastric cancer by upregulation of Survivin depend on activating the wnt/ $\beta$-catenin pathway. Exp Cell Res 379: 83-91, 2019.

12. Yuan LJ, Li JD, Zhang L, Wang JH, Wan T, Zhou Y, Tu H, Yun JP, Luo RZ, Jia WH and Zheng M: SPAG5 upregulation predicts poor prognosis in cervical cancer patients and alters sensitivity to taxol treatment via the mTOR signaling pathway. Cell Death Dis 5: e1247, 2014.

13. Abdel-Fatah TMA, Agarwal D, Liu DX, Russell R, Rueda OM, Liu K, Xu B, Moseley PM, Green AR, Pockley AG, et al: SPAG5 as a prognostic biomarker and chemotherapy sensitivity predictor in breast cancer: A retrospective, integrated genomic, transcriptomic, and protein analysis. Lancet Oncol 17: 1004-1018, 2016

14. Wang T, Li K, Song H, Xu D, Liao Y, Jing B, Guo W, Hu M, Kuang Y, Sun B, et al: p53 suppression is essential for oncogenic SPAG5 upregulation in lung adenocarcinoma. Biochem Biophys Res Commun 513: 319-325, 2019.

15. Li A, Wang Q, He G, Jin J and Huang G: DEP domain containing 1 suppresses apoptosis via inhibition of A20 expression, which activates the nuclear factor $\kappa \mathrm{B}$ signaling pathway in HepG2 cells. Oncol Lett 16: 949-955, 2018.

16. Livak KJ and Schmittgen TD: Analysis of relative gene expression data using real-time quantitative PCR and the 2(-Delta Delta C(T)) method. Methods 25: 402-408, 2001.

17. Tang Z, Li C, Kang B, Gao G, Li C and Zhang Z: GEPIA: A web server for cancer and normal gene expression profiling and interactive analyses. Nucleic Acids Res 45: W98-W102, 2017.

18. Rhodes DR, Yu J, Shanker K, Deshpande N, Varambally R, Ghosh D, Barrette T, Pandey A and Chinnaiyan AM: ONCOMINE: A cancer microarray database and integrated data-mining platform. Neoplasia 6: 1-6, 2004.

19. Okayama H, Kohno T, Ishii Y, Shimada Y, Shiraishi K, Iwakawa R, Furuta K, Tsuta K, Shibata T, Yamamoto S, et al: Identification of genes upregulated in ALK-positive and EGFR/KRAS/ALK-negative lung adenocarcinomas. Cancer Res 72: 100-111, 2012.
20. Sanchez-Vega F, Mina M, Armenia J, Chatila WK, Luna A, La KC, Dimitriadoy S, Liu DL, Kantheti HS, Saghafinia S, et al: Oncogenic signaling pathways in the cancer genome atlas. Cell 173: 321-37.e10, 2018.

21. Uhlen M, Zhang C, Lee S, Sjöstedt E, Fagerberg L, Bidkhori G, Benfeitas R, Arif M, Liu Z, Edfors F, et al: A pathology atlas of the human cancer transcriptome. Science 357: eaan2507, 2017.

22. Győrffy B, Surowiak P, Budczies J and Lánczky A: Online survival analysis software to assess the prognostic value of biomarkers using transcriptomic data in non-small-cell lung cancer. PLoS One 8: e82241, 2013.

23. Välk K, Vooder T, Kolde R, Reintam MA, Petzold C, Vilo J and Metspalu A: Gene expression profiles of non-small cell lung cancer: Survival prediction and new biomarkers. Oncology 79: 283-292, 2010.

24. Hanahan D and Weinberg RA: Hallmarks of cancer: The next generation. Cell 144: 646-674, 2011.

25. Levine B and Kroemer G: Biological functions of autophagy genes: A disease perspective. Cell 176: 11-42, 2019.

26. White E: The role for autophagy in cancer. J Clin Invest 125 : 42-46, 2015.

27. Song L, Dai Z, Zhang S, Zhang H, Liu C, Ma X, Liu D, Zan Y and Yin X: MicroRNA-1179 suppresses cell growth and invasion by targeting sperm-associated antigen 5-mediated Akt signaling in human non-small cell lung cancer. Biochem Biophys Res Commun 504: 164-170, 2018.

28. Wang L, Cao L, Wen C, Li J, Yu G and Liu C: LncRNA LINC00857 regulates lung adenocarcinoma progression, apoptosis and glycolysis by targeting miR-1179/SPAG5 axis. Hum Cell 33: 195-204, 2020 .

This work is licensed under a Creative Commons Attribution-NonCommercial-NoDerivatives 4.0 International (CC BY-NC-ND 4.0) License. 